\title{
Intrathoracic mass in a male Sprague-Dawley rat
}

\author{
Michael Hart, DVM, MS, Diplomate ACLAM ${ }^{1}$, Theresa M. Albers, DVM, Diplomate ACVP2 \& Diana Gettys, DVM ${ }^{3}$
}

An animal care technician at Georgia State University noticed that a 21-month-old, male Sprague-Dawley rat had an unkempt hair coat and a moderately thin body condition. The rat had been purchased from Charles River Laboratories (Crl:SD) and was being used in an IACUC-approved study but had received no experimental manipulations or treatments.

The rat was maintained in a standard polycarbonate shoebox cage with a wire bar top and corncob bedding. A microisolater was not used with the cage. Municipal tap water was provided ad libitum in a water bottle that was changed twice weekly. The light:dark cycle was 12-h:12-h. Technicians supplied the rat with 2014 Harlan Teklad Global 14\% Protein Rodent Maintenance Diet as feed.

A veterinarian examined the rat after the technician noted its condition. Upon manual restraint, the rat became dyspneic, and so it was immediately returned to its home cage before the veterinarian continued the examination. The difficulty in breathing did not readily resolve when the rat was returned to its home cage. Instead, the rat remained in a distressed condition with labored breathing for several minutes. The veterinarian decided to euthanize the rat. The rat was placed under deep sodium pentobarbital anesthesia, and the researchers who owned the rat carried out transcardial perfusion ( $0.9 \%$ saline immediately followed

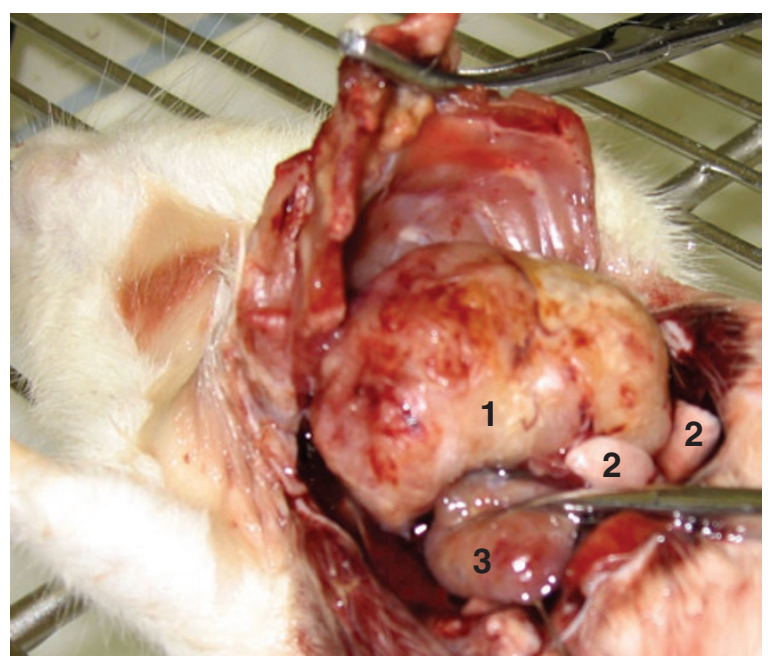

FIGURE 1 | An intrathoracic mass in a rat. The large tan mass with a mottled red surface (1) fills the thoracic cavity, causing caudal and lateral displacement of the lungs (2) and heart (3).

by $4 \%$ paraformaldehyde $/ 2.5 \%$ acrolein) to collect tissues required in the study protocol.

A necropsy carried out at the same time showed a $2-\mathrm{cm} \times 3-\mathrm{cm} \times 2-\mathrm{cm}$ firm, tanred mass with an irregular surface in the mediastinal region of the thoracic cavity. The mass created a substantial spaceoccupying lesion and displaced the lung lobes and heart caudally and laterally (Fig. 1). There was no gross evidence of local invasiveness by the mass. No other lesions were seen during the necropsy. The mass was fixed in 10\% neutral buffered formalin and, along with other tissues, submitted to Charles River Laboratories for histopathological assessment.

What are your differential diagnoses for this large, space-occupying mass in the thoracic cavity? Would the presence of this mass alone account for the dyspnea, or would you look at other organs histologically for abnormalities? How common is this type of lesion in rats?

What's your diagnosis?

\footnotetext{
${ }^{1}$ Georgia State University, Atlanta, GA. ${ }^{2}$ Charles River Laboratories, Boston, MA. ${ }^{3}$ Research Animal Diagnostic and Investigative Laboratory, University of Missouri, Columbia, MO. Correspondence should be addressed to M.H. (reomwh@langate.gsu.edu).
} 